
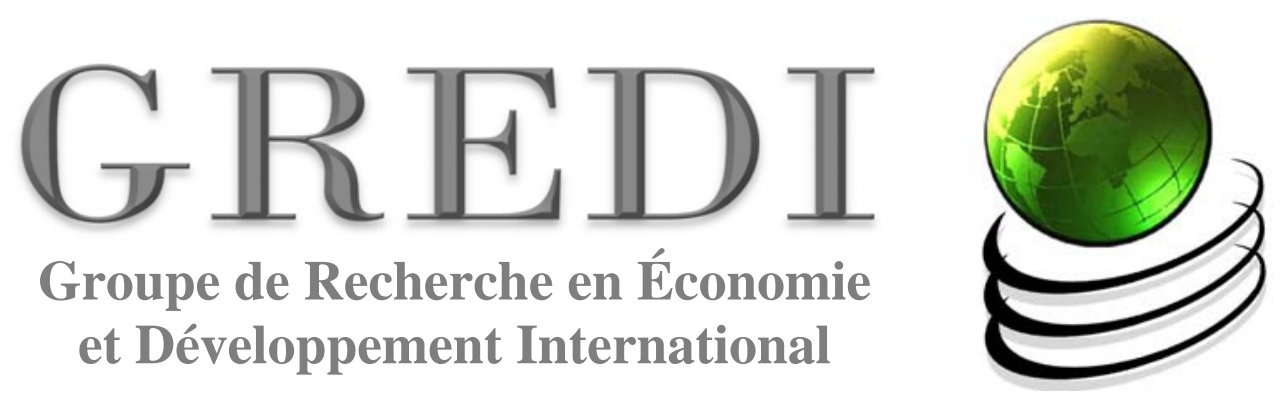

\author{
Cahier de Recherche / Working Paper \\ $10-18$
}

Quadratic Pen's Parade and the Computation of the Gini index

\author{
Stéphane Mussard, \\ Jules Sadefo Kamdem \\ Françoise Seyte \\ Michel Terraza
}




\title{
Quadratic Pen's Parade and the Computation of the Gini index
}

\author{
Stéphane Mussard \\ Université Montpellier I \\ LAMETA \\ Françoise Seyte \\ LAMETA \\ Université Montpellier I
}

\author{
J.Sadefo Kamdem * \\ LAMETA \\ Université Montpellier I \\ Michel Terraza \\ LAMETA \\ Université Montpellier I
}

\begin{abstract}
Following Milanovic's (1997) paper [Economics Letters, vol. 56, p. 4549], we propose a simple way to compute the Gini index when income $y$ is a quadratic function of its rank among $n$ individuals.
\end{abstract}

Key-words and phrases: Gini, Income inequality, Quadratic parade, Ranks.

JEL Classification: D63, D31, C15.

\footnotetext{
${ }^{*}$ Corresponding author: Université de Montpellier I, UFR Sciences Economiques, Avenue Raymond Dugrand - Site Richter - CS 79606, F-34960 Montpellier Cedex 2, France; E-mail: sadefo@lameta.univ-montp1.fr
} 


\section{Introduction}

Suppose that positive incomes, expressed as a vector $y$, depend on individuals' ranks $r_{y}$ in any given income distribution of size $n$. Suppose that incomes are ranked by ascending order and let $r_{y}=1$ for the poorest individual and $r_{y}=n$ for the richest one. Hence, following Lerman and Yitzhaki (1984), the Gini index may be rewritten as follows:

$$
G=\frac{2 \operatorname{cov}\left(y, r_{y}\right)}{n \bar{y}} .
$$

Here, $\operatorname{cov}\left(y, r_{y}\right)$ represents the covariance between incomes and ranks and $\bar{y}$ the mean income. It is straightforward to rewrite (1) as:

$$
G=\frac{2 \sigma_{y} \sigma_{r_{y}} \rho\left(y, r_{y}\right)}{n \bar{y}}
$$

where $\rho\left(y, r_{y}\right)$ is Pearson's correlation coefficient between incomes $y$ and individuals' ranks $r_{y}$, where $\sigma_{y}$ is the standard deviation of $y$ and where $\sigma_{r_{y}}$ is the standard deviation of $r_{y}$.

Following (2) and under the assumption of a linear Pen's parade (i.e. $y=a+b r_{y}$ ), Milanovic (1997) demonstrates that for a sufficiently large $n$, the Gini index can be further expressed as:

$$
G=\frac{\sigma_{y}}{\sqrt{3} \bar{y}} \rho\left(y, r_{y}\right)
$$

Milanovic's result is very interesting since it yields a simple way to compute the Gini index. However, as mentioned by Milanovic (1993) himself, "in almost all real world cases, Pen's parade is convex: incomes at first rise very slowly, and then their absolute increase, and finally even the rate of increase, accelerates". Thus, $\rho\left(y, r_{y}\right)$ which measures linear correlation will be less than 1. Again, from Milanovic (1993), a convex Pen's parade may be derived from a linear Pen's parade throughout regressive transfers (poorto-rich income transfers). Inspired from Milanovic's finding, we demonstrate in the sequel, without taking recourse to regressive transfers, that the Gini index can be computed with a quite general quadratic Pen's parade.

\section{Simple Gini Index with Quadratic Pen's Parade}

Consider a quadratic relation between incomes and ranks:

$$
y=a+b r_{y}+c r_{y}^{2} .
$$


The covariance between $y$ and $r_{y}$ is given by:

$$
\operatorname{cov}\left(y, r_{y}\right)=b \operatorname{cov}\left(r_{y}, r_{y}\right)+c \operatorname{cov}\left(r_{y}^{2}, r_{y}\right)=b \sigma_{r_{y}}^{2}+c \operatorname{cov}\left(r_{y}^{2}, r_{y}\right) .
$$

The mean income $\bar{y}$ is then:

$$
\bar{y}=a+b \overline{r_{y}}+c \overline{r_{y}^{2}}=a+\frac{1}{n} \sum_{i=1}^{n}\left[b i+c i^{2}\right]=a+b \frac{n+1}{2}+c \frac{(n+1)(2 n+1)}{6} .
$$

\subsection{The coefficient of variation}

Since incomes $y$ are positive, we use (1) by assuming that $c>0$ and $b^{2}-4 a c<$ 0 . We are now able to compute the coefficient of variation of incomes as follows:

$$
\frac{\sigma_{y}}{\bar{y}}=\frac{\sqrt{b^{2} \sigma_{r_{y}}^{2}+c^{2} \sigma_{r_{y}^{2}}^{2}+2 b c \operatorname{cov}\left(r_{y}, r_{y}^{2}\right)}}{a+b \frac{(n+1)}{2}+c \frac{(n+1)(2 n+1)}{6}} .
$$

The standard deviation of $r_{y}^{2}$ is:

$$
\begin{aligned}
\sigma_{r_{y}^{2}} & =\sqrt{\frac{1}{n} \sum_{i=1}^{n} i^{4}-\left(\frac{1}{n} \sum_{i=1}^{n} i^{2}\right)^{2}} \\
& =\sqrt{\frac{(n+1)(2 n+1)\left(3 n^{2}+3 n-1\right)}{30}-\frac{(n+1)^{2}(2 n+1)^{2}}{36}} \\
& =\frac{1}{6} \sqrt{\frac{(2 n+1)(n+1)\left(8 n^{2}+3 n-11\right)}{5}} .
\end{aligned}
$$

The standard deviation of $r_{y}$ is:

$$
\begin{aligned}
\sigma_{r_{y}} & =\sqrt{\frac{1}{n} \sum_{i=1}^{n} i^{2}-\left(\frac{1}{n} \sum_{i=1}^{n} i\right)^{2}} \\
& =\sqrt{\frac{(n+1)(2 n+1)}{6}-\frac{(n+1)^{2}}{4}} \\
& =\sqrt{\frac{n^{2}-1}{12}} .
\end{aligned}
$$


The covariance between $r_{y}$ and $r_{y}^{2}$ is:

$$
\begin{aligned}
\operatorname{cov}\left(r_{y}, r_{y}^{2}\right) & =\frac{1}{n} \sum_{i=1}^{n} i^{3}-\left(\frac{1}{n} \sum_{i=1}^{n} i\right)\left(\frac{1}{n} \sum_{i=1}^{n} i^{2}\right) \\
& =\frac{n(n+1)^{2}}{4}-\frac{n+1}{2} \frac{(n+1)(2 n+1)}{6} \\
& =\frac{n(n+1)^{2}}{4} \frac{n-1}{3} .
\end{aligned}
$$

Thereby, the coefficient of variation is expressed as:

$$
\begin{aligned}
\frac{\sigma_{y}}{\bar{y}} & =\frac{\sqrt{\frac{b^{2}}{36} \frac{3\left(n^{2}-1\right)}{12}+\frac{c^{2}}{36} \frac{(2 n+1)(n+1)\left(8 n^{2}+3 n-11\right)}{5}+2 b c \frac{n(n+1)^{2}}{2} \frac{n-1}{3}}}{a+b \frac{(n+1)}{2}+c \frac{(n+1)(2 n+1)}{6}} \\
& =\frac{|c|}{c} \frac{\sqrt{\frac{b^{2}}{c^{2}} \frac{3\left(n^{2}-1\right)}{(2 n+1)(n+1)}+\frac{8 n^{2}+3 n-11}{5(2 n+1)(n+1)}+12 \frac{b}{c} \frac{n(n-1)}{(2 n+1)^{2}}}}{\frac{6 a}{c} \frac{1}{(2 n+1)(n+1)}+\frac{6 b}{c} \frac{1}{2 n+1}+1} .
\end{aligned}
$$

Assuming that $\max (a, b) \ll c$, since $c>0$, we deduce the following limit:

$$
\lim _{n \rightarrow \infty} \frac{\sigma_{y}}{\bar{y}}=\frac{2}{\sqrt{5}} .
$$

Therefore, when $n$ is sufficiently large, we have the following approximation for the coefficient of variation:

$$
\frac{\sigma_{y}}{\bar{y}} \approx \frac{2}{\sqrt{5}}
$$

Remark 2.1 Under the quadratic Pen's parade assumption, we obtain an approximation for the coefficient of variation valued to be $2 / \sqrt{5}$, while Milanovic (1997) obtained $1 / \sqrt{3}$ under the linear Pen's parade assumption.

On the other hand, following Milanovic (1997):

$$
\lim _{n \rightarrow \infty} 2 \frac{\sigma_{r_{y}}}{n}=\lim _{n \rightarrow \infty} \sqrt{\frac{n^{2}-1}{3 n^{2}}}=\frac{1}{\sqrt{3}} .
$$

The product of (14), (13) and $\rho\left(y, r_{y}\right)$ entails the following result:

Theorem 2.1 Under the assumption of a quadratic Pen's parade, i.e., $y=$ $a+b r_{y}+c r_{y}^{2}$, the Gini index approximation is:

$$
G \simeq \frac{2}{\sqrt{15}} \rho\left(y, r_{y}\right)
$$

if $n$ is sufficiently large, $\max (a, b) \ll c, c>0$ and $b^{2}-4 a c<0$. 


\subsection{Application}

Following Milanovic's data (1997), we obtain the following results:

\begin{tabular}{|c|c|c|c|c|}
\hline \hline Country (year) & $n$ & $\rho\left(y, r_{y}\right)$ & $G=\frac{2}{\sqrt{15}} \rho\left(y, r_{y}\right)$ & $G=\frac{1}{3} \rho\left(y, r_{y}\right)$ \\
\hline Hungary (1993; annual) & 22062 & 0.889 & 0.459 & 0.296 \\
\hline Poland (1993; annual) & 52190 & 0.892 & 0.461 & 0.297 \\
\hline Romania (1994; monthly) & 8999 & 0.863 & 0.446 & 0.288 \\
\hline Bulgaria (1994; annual) & 7195 & 0.889 & 0.459 & 0.296 \\
\hline Estonia(1995; quarterly) & 8759 & 0.871 & 0.450 & 0.290 \\
\hline UK (1986; annual) & 7178 & 0.815 & 0.148 & 0.095 \\
\hline Germany (1889; annual) & 3940 & 0.744 & 0.384 & 0.248 \\
\hline US (1991; annual) & 16052 & 0.892 & 0.181 & 0.117 \\
\hline Russia (1993-4; quarterly)) & 16356 & 0.812 & 0.419 & 0.271 \\
\hline Kyrgyzstan (1993; quarterly) & 9547 & 0.586 & 0.303 & 0.195 \\
\hline \hline
\end{tabular}

Remark 2.2 As can be seen in the previous Table, Milanovic's Gini index based on the linear Pen's parade underestimates the Gini index obtained under the quadratic Pen's parade.

\section{Concluding Remarks}

Following Milanovic (1997), we have proposed another simple way to calculate the Gini coefficient under the assumption of a quadratic Pen's parade.

Two immediate and practical implications result from this new Gini expression. First, the possibility to address a simplified signification test since our Gini index (as well as Milanovic's one) is based on Pearson's correlation coefficient. Thereby, testing for the Gini index signification reduces to testing for the signification of Pearson's correlation coefficient (up to the constant $2 / \sqrt{15})$. This test relies on the well-known Student statistics based on the hyperbolic tangent transformation. Second, estimating the coefficients $\hat{a}, \hat{b}$ and $\hat{c}$, e.g. with Yitzhaki's Gini regression analysis, enables a parametric Gini index to be obtained that depends on parameters reflecting the curvature of Pen's parade, which may be of interest when one compares the shape of two income distributions. 


\section{References}

[1] Milanovic B. 1997. A simple way to calculate the Gini coefficient, and some implications. Economics Letters 56, 45-49.

[2] Lerman R.I., Yitzhaki S., 1984. A note on the calculation and interpretation of the Gini index. Economics Letters 15, 363-368. 